\title{
A strategy for implementing differential VLBI
}

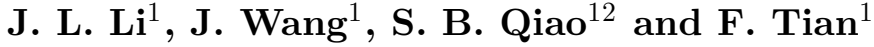 \\ ${ }^{1}$ Shanghai Astronomical Observatory, Shanghai 200030, China \\ ${ }^{2}$ Surveying and Mapping Institute, Zhengzhou 450052, China \\ email: jll@shao.ac.cn
}

\begin{abstract}
Based on past astrometric and geodetic VLBI observations, a strategy for implementing differential VLBI (DVLBI) is developed by interpolating the non-geometric delay (NGD) at the target's direction using observations of several reference sources spreaded out within a circular ring centered on the target. In contrast to the ordinary approach, in our design the limitations in the angular distance are relaxed and the effects of observational uncertainties in reference sources are reduced. Analysis shows that in our design a precision of the NGD correction in S-band reaches about 1ns only. Our design can be adopted for observations of weak sources and in deep space exploration.
\end{abstract}

Keywords. atmospheric effects, methods: data analysis, reference systems, astrometry

\section{Introduction}

In order to retain high precision in relative position determination by DVLBI, the angular distance between the reference and target objects should be very small. For single and dual-frequency observations it is usually less than $1^{\circ}$ and $5^{\circ}$ respectively (Sovers et al. (1998)). There are about several hundreds of sources with high position precision, or about one source per hundred square degrees. In practice a suitable reference source may not be available, thus preventing DVLBI from being widely applied. We developed an implementation strategy for DVLBI by extending the angular distance limitation in order to make this technique more easily applicable.

In the NGD, the clock bias and the instrument delay are relatively stable within a short period of time. They shift the NGD distribution camber rather than change the shape. The ionosphere delay is related to the frequency and direction. At $8.4 \mathrm{GHz}$ the zenith delay ranges from $0.03 \mathrm{~m}$ to $0.6 \mathrm{~m}$. The troposphere delay is mainly dependent on the elevation. It is about $2 \mathrm{~m}$ at the zenith and $20 \mathrm{~m}$ at $6^{\circ}$. From the magnitudes and characteristics of all components that shape the NGD distribution camber, we conclude that it is mainly affected by the troposphere delay. Simulations and checks by real observations show that, for a given baseline and observations made within two hours, the NGD distribution camber is smooth, which opens up the possibility for NGD interpolation.

\section{A strategic design for the NGD interpolation}

For a given baseline and observations made within a short period of time, the NGD can be described as a scalar field on the surface of a half sphere centered at the reference station. Imagine that, for this scalar field with smooth change, the field values at all the points on a great circle passing through target $T$ form a data series. There are various great circles passing through $T$, which associated with various data series. However, the series value at $T$ for all the series should be equal within the range of uncertainty. Based 

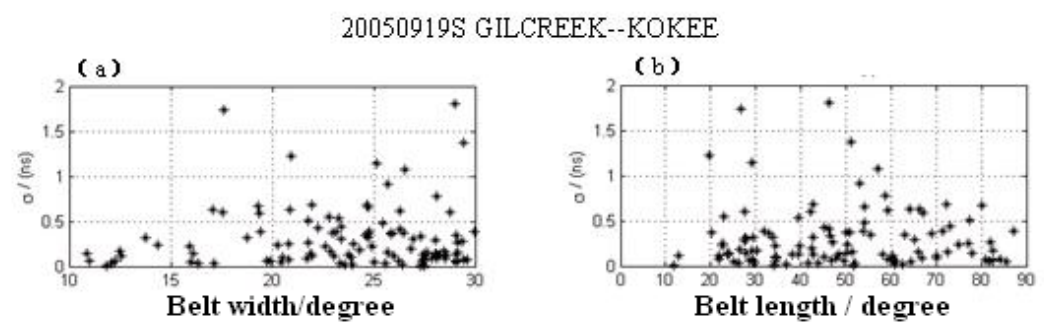

Figure 1. The distribution of $\sigma$ versus the width and length of the sample ring

on this imagination and in order to sustain sufficient data points in the case of radio sources, a ring with some width and centered along a great circle passing through $T$ could be marked. Neglecting the ring width, and counting from one end, let the accumulated sum of the arc-lengths between neighboring points be the indices of the data series. By smoothing and interpolating on the data series the NGD at $T$ will be resulted. Based on the archive observations of VLBI experiments the following strategy is proposed. (1) Plot the NGD data of a baseline within two hours on the topocentric spherical plane of the reference station. (2) On the plot mark down some great circle rings with some widths. Let each ring contain as many points as possible. Take all the points on a ring as an analysis collection. (3) Assuming a collection contains $N$ sources, perform a poly-fit to the data and the smoothed NGD at each point is obtained as $\tau_{s}$. (4) Take source 3 through $N-2$ respectively as target and all the rest as references, thus resulting in $N-4$ analytical samples. Apply a poly-fit to all the NGD data of references in a sample and then interpolate the NGD at the target according to the arc-length index, taken as $\tau_{i}$. (5) Take the difference between the corresponding interpolated and smoothed NGD as $\nu_{i m s}=\tau_{i}-\tau_{s}$. For a collection of $N$ sources, $N-4$ values of $\nu_{i m s}$ will result. Define the standard deviation of $\nu_{i m s}$ as $\sigma=\left(\sum \nu_{i m s}^{2} /(N-5)\right)^{1 / 2}$, and let $\sigma$ be the criteria for evaluating the NGD interpolation.

\section{Check by real observations and discussion}

It is easy to understand that the precision of interpolation of the NGD for the target depends on the number of reference sources, their distribution, and observational uncertainties. Figure 1 shows a check on real NGD observations in the $S$ band for baseline Gilcreek-Kokee on September 9 of 2005. It is the distribution of $\sigma$ versus the width and length of the ring when the number of sources in each sample is no less than 6 . It is shown that when the width and length of the ring are restricted within $30^{\circ}$ and $90^{\circ}$ respectively, most of the points are well below 1 ns.

Numerous VLBI experiments have been checked and the situation is similar to Fig. 1. Compared to ordinary DVLBI, in our design the limitation in the angular distance is relaxed and the observation uncertainties of references are reduced. Our design can be adopted in observations of weak sources and in deep space exploration.

\section{Acknowledgements}

This work is supported by NSFC (No. 10778635, No. 10173019, No. 10473019), Chinese lunar exploration project CE-1 and STC of Shanghai Municipality (06DZ22101).

\section{References}

Sovers, O. J., Fanselow, J. L., \& Jacobs, C. S. 1998, Reviews of Modern Physics 70, 1393 